\title{
Deuda de los centros de conciliación de los consultorios jurídicos con las personas en situación de insolvencia. Siete años de inactividad del procedimiento de insolvencia en escenarios conciliatorios de práctica legal
}

\author{
The Debt of Legal Clinics' Conciliation Centers \\ with Insolvent Individuals. Seven Years' \\ Inactivity of the Insolvency Procedure in \\ Conciliatory Events of Legal Practice
}

Dívida dos centros de conciliação dos consultórios jurídicos com as pessoas em situação de inadimplência. Sete anos de inatividade do procedimento de inadimplência em cenários conciliatórios de prática legal

Juan Carlos Montoya* 


\section{Resumen}

En Colombia, desde el año 2012, se definió un procedimiento de insolvencia para personas naturales no comerciantes cuyo propósito es la estabilización financiera del deudor y la recuperación del crédito a favor de sus acreedores. Según el marco normativo, este procedimiento podría iniciarse gratuitamente ante centros de conciliación de los consultorios jurídicos de las facultades de derecho. A pesar de lo anterior, y aun cuando existe la imperiosa necesidad de atención y adelantamiento del procedimiento para las personas en condiciones de vulnerabilidad que se encuentran en situación de insolvencia, al año 2019 y habiendo pasado 7 años desde la expedición de dicho procedimiento, ningún centro de conciliación universitario de la ciudad de Bogotá estaba prestando este servicio. El presente artículo es resultado de un ejercicio investigativo cuyo propósito consistió en determinar los factores que explicarían lo anterior. De esta manera, se relaciona el concepto de pobreza y precariedad económica con la situación de insolvencia; se desarrollan los propósitos del procedimiento de insolvencia de persona natural no comerciante y la situación de debilidad manifiesta de los deudores insolventes en Colombia; se hace referencia a los propósitos pedagógicos y sociales de los consultorios jurídicos, y se exponen los resultados.

\section{Palabras clave}

Insolvencia; crisis financiera personal; persona natural no comerciante; consultorios jurídicos; servicios legales gratuitos; educación jurídica experiencial. 


\section{Abstract}

Since 2012, Colombia has a defined insolvency procedure for uncommercial individuals whose purpose is to stabilize the debtor financially and recover the debt for creditors. According to the regulatory framework, this procedure may be initiated free of charge with conciliation centers of legal clinics. However, and even though there is an urgent need for counseling and application of the procedure for insolvent people in vulnerable conditions, no university conciliation center in Bogotá provides this service as of 2019, seven years after the issuance of this procedure. This article derives from a research exercise whose aim is to determine the factors that would explain this situation. For this, we relate the concepts of poverty and economic precariousness to insolvency, elaborate on the purposes of the insolvency procedure for uncommercial individuals and the manifest weakness of insolvent debtors in Colombia, refer to the pedagogical and social objectives of legal clinics, and present the results.

\section{Keywords}

Insolvency; personal financial crisis; uncommercial individual; legal clinics; free legal services; experiential law education. 


\section{Resumo}

Na Colômbia, desde 2012, foi definido um procedimento de inadimplência para pessoas físicas não comerciantes cujo objetivo era a estabilização financeira do devedor e a recuperação do crédito a favor de seus acredores. Segundo a norma, esse procedimento poderia ser iniciado de forma gratuita nos centros de conciliação dos consultórios jurídicos das faculdades de Direito. Apesar disso e ainda quando existe a imperiosa necessidade de atenção e realização do procedimento para as pessoas em condições de vulnerabilidade que se encontram em situação de insolvência, em 2019, sete anos da expedição do procedimento, nenhum centro de conciliação universitário da cidade de Bogotá está prestando esse serviço. Este artigo é resultado de um exercício investigativo cujo objetivo foi determinar os fatores que explicariam a situação. Assim, é relacionado o conceito de pobreza e precariedade econômica com a situação de inadimplência; são desenvolvidos os objetivos do procedimento de inadimplência de pessoa física não comerciante e a situação de debilidade manifestada dos devedores inadimplentes na Colômbia; é feita referência aos propósitos pedagógicos e sociais dos consultórios jurídicos, e são expostos os resultados.

\section{Palavras-chave}

Inadimplência; crise financeira pessoal; pessoa física não comerciante; consultórios jurídicos; serviços legais gratuitos; educação jurídica experiencial. 
Sumario: 1 Vulnerabilidad económica e insolvencia. 2. El procedimiento de insolvencia de persona natural no comerciante; propósitos. 3. La prestación del procedimiento de insolvencia. 4. Estado actual de los prestadores de servicios legales gratuitos que adelantan el procedimiento de insolvencia de persona natural no comerciante en Bogotá. 5. Educación jurídica experiencial e insolvencia. 6. Factores. Conclusiones. Bibliografía.

\section{Vulnerabilidad económica e insolvencia}

Según Spicker et al., la pobreza, en un sentido material, se define como un fenómeno que se presenta cuando la población no tiene algo que necesita, o cuando carece de recursos para acceder a las cosas que requiere. ${ }^{1}$ En la misma línea, Baratz y Grigsby plantean el concepto de pobreza como una privación severa de bienestar físico y bienestar mental, estrechamente asociada con inadecuados recursos económicos y consumos, ${ }^{2}$ definiciones que plantean la carencia de medios, lo cual imposibilita a la persona a acceder a bienes y servicios que le provean bienestar.

Según lo informado en Portafolio, el Departamento Administrativo Nacional de Estadística (DANE) estimó que 12,8 millones de personas se encuentran en condiciones de pobreza monetaria, ${ }^{3}$ es decir, el $26 \%$ de los colombianos tienen ingresos por debajo del monto mínimo mensual establecido como necesario para cubrir necesidades básicas. Así mismo, el Observatorio Laboral de la Universidad del Rosario indicó que el $25 \%$ de los ocupados asalariados en el país gana exactamente un salario mínimo. ${ }^{4}$

Los datos anteriores, muestran cómo un grueso importante de la población colombiana se encuentra en condiciones económicas precarias. En todo caso, operaciones que suponen asumir obligaciones dinerarias son el día a día para millones de colombianos, pues, la necesidad de cubrir necesidades impostergables e inmediatas es suplida, en su mayoría, a través de la adquisición de préstamos de dinero que permitan solventarlas.

Así, en la actualidad, millones de ciudadanos se encuentran en niveles altísimos de endeudamiento como resultado de la adquisición de obligaciones que exceden su capacidad de pago, lo que genera situaciones de inestabilidad financiera que los llevan a incumplimientos graves de sus obligaciones.

Entonces, existe una relación estrecha entre las precarias condiciones económicas de las personas y la situación de cesación de pagos, es decir, no tener

AA.VV., Pobreza: un glosario internacional, Buenos Aires, Clacso, 2009, p. 292.

AA.VV., Thoughts on poverty and its elimination, Cambridge, Cambridge University Press, 1972, p. 120.

3 "Aún hay 3,5 millones de personas en pobreza extrema", Portafolio (18 de octubre de 2018), en https://www. portafolio.co/economia/aun-hay-3-5-millones-de-personas-en-pobreza-extrema-522423

4 AA.VV., Informe 4: Panorama de la negociación del salario mínimo 2018, Bogotá, Observatorio Laboral de la Universidad del Rosario, 2018. 
recursos suficientes implica indefectiblemente, en la mayoría de casos, incurrir en el incumplimiento de obligaciones por parte de los deudores frente a sus acreedores.

Entre diversos factores, la escasa educación sobre conceptos financieros, la deficiente administración y falta de conciencia sobre las finanzas personales, el desempleo, la carencia de oportunidades que reporten ingresos, el sobreendeudamiento y, claramente, la carencia de recursos, son causas que llevan a las personas a sucumbir en crisis financieras que imposibilitan el cumplimiento de sus obligaciones.

La académica española Ana Alemán Monterreal, en su libro La insolvencia: una cuestión de terminología jurídica, señala que la insolvencia, como concepto jurídico, ha sido definida de manera relativamente heterogénea cuando una persona carece de suficiencia patrimonial, o se encuentra en incapacidad de pagar sus obligaciones cuando se consideran ilíquidas, sobreendeudadas, o cuando reporta activos inferiores al pasivo de su patrimonio, es decir, existe una idea constante vinculada a una situación económica en la que se encuentra el deudor que no puede satisfacer a su acreedor, lo que provoca consecuentemente, una imposibilidad de pago que origina una lesión del derecho de crédito con el consiguiente nacimiento de una responsabilidad del deudor o de un periculum en el sentido de riesgo para el acreedor. ${ }^{5}$

\section{El procedimiento de insolvencia de persona natural no comerciante; propósitos}

Como forma de respuesta a las situaciones de insolvencia, en diversos ordenamientos jurídicos del globo se han establecido procedimientos concursales aplicables a las personas que se encuentran en dicha situación. Para el caso particular de Colombia, se definió un procedimiento que posibilita la estabilización financiera de los deudores, con el fin de facilitar salidas válidas a su estado de crisis económica personal. Así, desde el año 2012, con la expedición del Código General del Proceso, se estructuró el proceso de insolvencia de persona natural no comerciante como régimen jurídico que permite que deudores insolventes, que sean personas naturales, y las cuales no detenten la calidad de comerciantes, inicien un procedimiento que en principio busca la negociación de las deudas a través de un acuerdo con sus acreedores con la finalidad de normalizar las relaciones crediticias, así como la convalidación de acuerdos o, en últimas, su liquidación patrimonial.

5 Ana Alemán Monterreal, La insolvencia: una cuestión de terminología jurídica, Madrid, Andavira Editora, 2010. 
Esta oportunidad de salida a la crisis financiera le permite inicialmente al deudor sentarse ordenadamente con todos sus acreedores para que, contando con la mediación de un conciliador, exponga una propuesta de pago de las obligaciones; los acreedores tienen la posibilidad de formular contrapropuestas con el fin de lograr acuerdos serios que permitan, por un lado, la recuperación del crédito a favor del acreedor, y, por otro, la estabilización financiera del deudor bajo condiciones ajustadas a su capacidad de pago.

De esta manera, la etapa inicial del procedimiento busca organizar un escenario de negociación de deudas como oportunidad de deliberación razonada entre el deudor y sus acreedores, el cual resulta fundamental para el alcance de la finalidad de este procedimiento de estabilización económica, tan necesario en un contexto como el colombiano.

Este régimen de insolvencia aplicable a las personas naturales que no son comerciantes, el cual se encuentra fijado en los artículos 521 al 576 del Código General del Proceso, ${ }^{6}$ se dio a partir de una exhortación hecha por la Corte Constitucional ${ }^{7}$ al legislador en la que el alto tribunal indicó principalmente que los procedimientos concursales son mecanismos orientados a la protección del crédito y, así mismo, permiten la realización del principio de solidaridad en aquellos casos en que como consecuencia de una situación de insolvencia, el deudor se encuentra en un estado de debilidad manifiesta que afecta sus derechos fundamentales $\mathrm{y}$, por tanto, resulta acorde con dicho principio que se diseñare un proceso con el propósito de lograr el rescate financiero de los deudores.

Sobre la situación de debilidad manifiesta que se predica de ciertas personas en Colombia, la Constitución Política indica que "el Estado protegerá especialmente a aquellas personas que, por su condición económica, física o mental, se encuentren en circunstancia de debilidad manifiesta y sancionará los abusos o maltratos que contra ellas se cometan". ${ }^{8}$ Luego, los deudores insolventes se encuentran en esta situación por razón de una crisis económica estructural que los ha llevado a incumplir sistemáticamente con sus obligaciones, por lo que deben gozar de una protección especial por expresa disposición de rango constitucional.

Ley 1564 de 2012, 12 de julio. Diario Oficial 48.489.

La Corte Constitucional de Colombia, en Sentencia C-699 de 2007, señaló que: "Si bien los procesos concursales son, fundamentalmente, mecanismos orientados a la protección del crédito, no es menos cierto que a través de ellos puede hacerse efectivo el principio de solidaridad en aquellos casos en los que, como consecuencia de una situación de insolvencia, el deudor se encuentre en una situación de debilidad manifiesta que afecte sus derechos fundamentales, razón por la cual resultaría acorde con dicho principio que el legislador estableciese un proceso concursal específico para las personas naturales no comerciantes que se encuentren en un estado de insolvencia. Para tal efecto, la Corte hará un exhorto al Congreso de la República, para que dentro de su potestad de configuración legislativa expida un régimen universal al que puedan acogerse las personas naturales no comerciantes en situación de insolvencia". 


\section{La prestación del procedimiento de insolvencia}

Esta circunstancia de debilidad manifiesta por razones económicas debe dar lugar a la aplicación del principio de solidaridad como uno de los criterios en que se inspira el procedimiento de insolvencia de persona natural no comerciante en Colombia, pues, resulta claro que no tener capacidad para soportar las cargas financieras puede dar lugar a la configuración de una situación que estructuralmente afecte los derechos fundamentales del deudor y de los miembros de su núcleo familiar, lo cual demanda un despliegue no solo a cargo del Estado, sino incluso de los particulares en atención a los deberes que se exigen a los distintos componentes de la sociedad con el ánimo de lograr los fines esenciales de la organización política.

Al ser la solidaridad un valor fundante del Estado social de derecho, en el actual régimen de insolvencia de persona natural no comerciante se determinó que la iniciación del procedimiento para la negociación de deudas o convalidación de acuerdos ante centros de conciliación de facultades derecho y de las entidades públicas será gratuito. Así mismo, la norma señala que los centros de conciliación privados y las notarías podrán cobrar por estos servicios, y que el gobierno nacional determinará las tarifas que podrían ser aplicadas en estos centros de conciliación privados y notarias a las personas insolventes por dar inició al trámite del procedimiento.

Así, se precisa que las organizaciones ante las cuales se puede iniciar este procedimiento de rescate financiero pueden ser los centros de conciliación de facultades de derecho o de entidades públicas, en las cuales el trámite sería gratuito. Así mismo, se podrá optar por iniciar el trámite del procedimiento ante centros de conciliación privados y notarias, con un valor a cargo del deudor insolvente.

De esta manera, resulta complejo y hasta contradictorio ofrecerle al deudor insolvente como oportunidad de recuperación financiera la iniciación de un procedimiento para acceder al cual debe pagar una tarifa. Lo anterior configura una barrera relevante que limita el inicio del procedimiento para la recuperación económica del deudor, quien tiene restringidos los medios para conseguir el dinero que le permita iniciar este trámite. Por ello la importancia y necesaria oportunidad de trámite del procedimiento de manera gratuita ante centros de conciliación de facultades derecho y de entidades públicas en un país como Colombia, en el que la prestación de servicios legales gratuitos resulta sumamente necesaria respecto a la población que se encuentra en condiciones de vulnerabilidad económica y social, de ahí la gran importancia de preservar dicha característica en ese contexto. 
Cabe señalar que los centros de conciliación, sean de facultades de derecho, o de entidades públicas o privadas, requieren de autorización por parte del Ministerio de Justicia para darle trámite a este procedimiento. En este entendido el Gobierno nacional, mediante decreto, estableció de manera precisa los requisitos que deben cumplir los centros de conciliación, a fin de obtener la autorización para el trámite del procedimiento de insolvencia de persona natural. ${ }^{9}$ Así, los centros de conciliación interesados en recibir autorización para conocer de los procedimientos de insolvencia deberán presentar ante el Ministerio de Justicia una solicitud en tal sentido suscrita por el representante legal de la entidad promotora del centro y reunir los siguientes requisitos:

Haber obtenido del Ministerio de Justicia y del Derecho autorización para su funcionamiento como centro de conciliación, como mínimo, tres (3) años antes de la radicación de la solicitud, y que dicha autorización no haya sido revocada;

Haber operado durante los tres (3) años anteriores a la radicación de la solicitud, y haber tramitado a lo largo de ellos no menos de cincuenta (50) casos de conciliación, según reporte generado por el Sistema de Información de la Conciliación;

No haber sido sancionado por el Ministerio de Justicia y del Derecho en los últimos tres (3) años;

Demostrar que cuenta con salas de audiencias para conciliación con una capacidad mínima de diez (10) personas;

Presentar una propuesta de modificación o adición a su Reglamento Interno, que incluya el procedimiento y los requisitos para integrar la lista de conciliadores en insolvencia de la persona natural no comerciante, en los términos establecidos en el presente decreto.

La citada disposición indica que el Ministerio de Justicia decidirá sobre la solicitud dentro de los sesenta días calendario siguientes a su presentación, dentro de los cuales podrá requerir al centro de conciliación o a la entidad promotora para que complete o adicione la documentación presentada con la solicitud.

Como se puede verificar, los requisitos para que un centro de conciliación de una facultad de derecho de una entidad pública o de un centro de conciliación privado sea habilitado para el trámite de este procedimiento no son de difícil cumplimiento y obedecen a criterios formales con la finalidad de garantizar un servicio idóneo y de calidad. 


\section{Estado actual de los prestadores de servicios legales gratuitos que adelantan el procedimiento de insolvencia de persona natural no comerciante en Bogotá}

Una vez aclarado que existe la posibilidad para que un deudor insolvente pueda buscar la oportunidad de rescate financiero ante una entidad que tramite el procedimiento de insolvencia de manera gratuita, se consultó con el Ministerio de Justicia sobre las entidades ubicadas en la ciudad de Bogotá que están autorizadas para prestar este servicio gratuito a la comunidad. Según lo reportado por la Dirección de Métodos Alternativos de Solución de Conflictos del Ministerio, en la ciudad de Bogotá no existe ningún centro de conciliación que realice este trámite gratuitamente, es decir, ningún centro de conciliación de consultorios jurídicos o de entidades públicas se encuentra autorizado por el Ministerio de Justicia para conocer de los procedimientos de insolvencia de persona natural no comerciante.

Según la respuesta del Ministerio de Justicia, hasta inicios de 2019 el único centro de conciliación de consultorio jurídico de la ciudad de Bogotá que había solicitado la habilitación para el trámite del procedimiento de insolvencia de persona natural, desde la expedición del decreto reglamentario de este procedimiento, fue el de la Universidad Nacional de Colombia (Sede Bogotá). No obstante, dicho centro de conciliación universitario, mediante solicitud posterior presentada por su representante legal, solicitó la revocación de la autorización, sin conocerse los motivos, es decir, ningún otro centro de conciliación de las facultades de derecho ubicadas en esta ciudad ha solicitado la autorización para poder tramitar este procedimiento, aun cuando este acto administrativo fue expedido en el año 2012.

Esta situación pone en evidencia una clarísima ausencia de voluntad para tramitar el procedimiento de insolvencia para personas naturales no comerciantes por parte de las entidades que deberían hacerlo gratuitamente en la ciudad de Bogotá, situación que genera preocupación por el grueso de ciudadanos que se encuentran en estas circunstancias de cesación de pago de sus obligaciones por estar atravesando una crisis económica estructural y quienes se hallan en la búsqueda de oportunidades de recuperación para solucionar su situación acudiendo a estas entidades.

Frente a este panorama particular e inverosímil, cabe preguntarse por los factores que explican por qué en la ciudad de Bogotá ningún centro de conciliación de facultad de derecho se ha habilitado para el trámite de este procedimiento. Más aún si se tiene en cuenta la densidad demográfica de la ciudad, de alrededor de ocho millones de habitantes, así como el elevado número de 
facultades de derecho en la ciudad que asciende a más de 40 según lo que reporta el Sistema Nacional de Información de la Educación Superior (SNIES) del Ministerio de Educación de Colombia ${ }^{10}$.

Respecto a lo anterior, se consultó al Ministerio de Justicia si existía una política pública en materia de acceso a la justicia para personas naturales no comerciantes en situación de insolvencia. Este Ministerio se limitó a responder que existe un marco normativo para las personas naturales no comerciantes contenido en el Título IV Capítulo III y IV de la Ley 1564 de 2012. En igual sentido, se consultó si existían, y cuáles eran, las estrategias o acciones para promover que los centros de conciliación de los consultorios jurídicos se habilitaran y tramitaran el procedimiento de insolvencia de persona natural no comerciante. Ante esta consulta la entidad señaló que la Dirección de Métodos Alternativos de Solución de Conflictos, mediante la Circular CIR18-0000074-DMA2100 del 3 de julio de 2018, convocó a los centros de conciliación de los consultorios jurídicos de universidades y de las entidades públicas para que solicitaran ante el Ministerio de Justicia la autorización para conocer los procedimientos de insolvencia de la persona natural no comerciante. A partir de dicha convocatoria se observó con preocupación que, a la fecha, existía una limitada oferta de centros de conciliación gratuitos autorizados para conocer dichos procedimientos de insolvencia, lo cual indica que en todo el país únicamente dos centros de conciliación se encuentran habilitados y prestan este servicio gratuitamente, los cuales se ubican en las ciudades de Cúcuta y Neiva.

Esta situación resulta sumamente alarmante, pues los centros de conciliación de los consultorios jurídicos de las facultades de derecho y de las entidades públicas tienen un compromiso social consistente en facilitar acceso a la justicia a población que se encuentra en condiciones de vulnerabilidad social y económica. Así las cosas, en Bogotá ninguna persona que se encuentre en los supuestos de insolvencia puede acceder al trámite de este procedimiento de manera gratuita en un centro de conciliación universitario ni de entidad pública.

La gratuidad de la administración de justicia ha sido desarrollada jurisprudencialmente como un principio cuya finalidad es garantizar un orden justo que haga realidad la igualdad jurídica y material para los ciudadanos. ${ }^{11}$ Parti-

10 El Sistema Nacional de Información de la Educación Superior (SNIES) ha sido creado por el Ministerio de Educación de Colombia para responder a las necesidades de información de la educación superior en el país. En este sistema se recopila y organiza la información relevante sobre la educación superior que permite hacer planeación, monitoreo, evaluación, asesoría, inspección y vigilancia del sector. Así, la información relacionada con las instituciones de educación superior y los programas académicos que estas ofrecen puede ser consultada en la página web del SNIES, en https://www.mineducacion.gov.co/sistemasinfo/SNIES/

11 La Corte Constitucional de Colombia, en la Sentencia T-522 de 1994, señaló: “De la Constitución se puede inferir el principio de gratuidad de la circunstancia de que la justicia constituye uno de los pilares o fundamentos esenciales para lograr la convivencia, la paz y un orden justo que haga realidad la igualdad jurídica y material, enmarcado dentro de la filosofía y el realismo del Estado Social de Derecho, justicia cuya aplicación, operatividad y eficacia se hace efectiva cuando las instituciones procesales creadas como instrumentos para 
cularmente, y como se señaló, en el trámite de este procedimiento se encuentra determinado, según el artículo 535 del Código General del Proceso, que la iniciación del mismo será gratuita ante centros de conciliación de consultorios jurídicos de facultades de derecho y de las entidades públicas.

\section{Educación jurídica experiencial e insolvencia}

Ahora bien, respecto a los consultorios jurídicos como espacios de educación experiencial, desde sus orígenes hace ya 48 años, estas instituciones se han establecido como escenarios que posibilitan la materialización del derecho al acceso a la justicia de un amplio número de personas en condiciones de vulnerabilidad social o económica por medio de la prestación de servicios legales gratuitos por estudiantes de los últimos semestres de la carrera de Derecho, quienes se encuentran bajo la supervisión de asesores docentes. ${ }^{12}$

El académico Daniel Bonilla señala que globalmente se ha configurado un concepto de los consultorios jurídicos como instituciones que, en su acepción transnacional estándar, persiguen dos objetivos principales: formar estudiantes que tengan las destrezas necesarias para actuar competente y éticamente en la práctica profesional y contribuir a elevar los niveles de justicia social de la comunidad política en la que están inmersos. ${ }^{13}$

En Colombia, los consultorios jurídicos son de obligatoria constitución en las facultades de derecho, y en estos los estudiantes de últimos semestres realizan ejercicios de práctica, utilizando el derecho como una herramienta de transformación social en la asistencia de casos de personas o grupos poblacionales en condiciones de vulnerabilidad. Así mismo, diferentes consultorios jurídicos universitarios han diversificado la práctica legal más allá del litigio, expandiendo sus actividades a la pedagogía de derechos, la incidencia política a favor de grupos históricamente discriminados, el desarrollo de ejercicios de comprensión y análisis de fenómenos sociales, entre otras actividades.

De esta manera, la conciencia jurídica en el país reconoce a los consultorios jurídicos como organizaciones ineludibles de una tradición de casi cinco décadas que hace parte significativa de la formación de los abogados y las abogadas en

asegurar su vigencia, arbitran los mecanismos idóneos para que puedan acceder a ellas todas las personas en condiciones de igualdad. La gratuidad es, en esencia, la condición para hacer realidad el acceso a la justicia en condiciones de igualdad, pues la situación económica de las partes no puede colocar a una de ellas en situación de privilegio frente a la otra ni propiciar, por consiguiente, la discriminación".

12 Los consultorios jurídicos se introdujeron en Colombia a través del Decreto Ley 196 de 1971, y su constitución es de obligatorio cumplimiento para las facultades de derecho oficialmente reconocidas.

13 Daniel Bonilla Maldonado, Abogados y justicia social: derecho de interés público y clínicas jurídicas, Bogotá, Ediciones Uniandes, Siglo del Hombre Editores, 2018. 
Colombia. En este sentido, los consultorios jurídicos de las facultades de derecho colaboran como prestadores de servicios legales gratuitos, respaldando el reclamo de acceso a la justicia de los miles de ciudadanos y ciudadanas que día a día necesitan de sus servicios para afrontar sus asuntos legales.

Infortunadamente, y dada la inexistencia de centros de conciliación adscritos a los consultorios jurídicos de facultades de derecho habilitados para el trámite del procedimiento de insolvencia en la ciudad de Bogotá, cuando un ciudadano recurre a una de estas instituciones en busca de auxilio con la idea de identificar oportunidades de recuperación financiera en razón de su crisis económica estructural, existiendo el procedimiento estatuido por la ley para darle trámite a su situación, a estos no se les adelanta el procedimiento, y se les indica la posibilidad de iniciarlo ante los centros de conciliación privados o notarias, entidades que cobran una tarifa por su adelantamiento.

Y es que son principalmente estas personas en condiciones de vulnerabilidad por características de orden económico, como lo es una crisis financiera, quienes recurren con regularidad ante estas instituciones buscando una oportunidad de ayuda; por esta razón, si las personas en situación de insolvencia no pueden acceder al procedimiento de manera gratuita a través de entidades como los centros de conciliación de las facultades de derecho, podría configurarse indefectiblemente una grave desprotección a nivel constitucional de sus derechos fundamentales en consideración a sus circunstancias de debilidad manifiesta por razones económicas, por lo que el deber de protección especial a cargo del Estado para con estas personas no se estaría cumpliendo dada la inexistencia de entidades que tramiten el procedimiento de manera gratuita, sumado a la ausencia de una política pública en materia de acceso a la justicia para personas naturales no comerciantes en situación de insolvencia.

De este modo, y a pesar de que existe un marco normativo suficiente que ofrece garantías para la solución de la situación de crisis del insolvente, si no existen instituciones que de manera gratuita ofrezcan dicho servicio a la población en condiciones de vulnerabilidad económica, la eficacia y validez del régimen jurídico se ve disminuida precisamente por estas circunstancias que, de facto, imposibilitan acceder al procedimiento de insolvencia al no poder ser iniciado dadas las limitaciones como el pago de la tarifa que podría cobrar un centro de conciliación privado o en una notaría.

Cabe preguntarse, entonces, ¿es eficaz la institución de un procedimiento de insolvencia a favor de personas naturales no comerciantes, cuando no existen entidades que tramiten dicho procedimiento de manera gratuita para quienes no tienen la capacidad económica de asumir el costo?

De acuerdo con Manuel María Zorrilla, la efectividad del derecho cobra sentido solo si hay verdaderas oportunidades de acceder a los beneficios que plantea 
la norma, y el derecho rinde un servicio auténtico a la comunidad y acredita su sincero propósito de tutela y de cambio. ${ }^{14}$

Bajo la situación que se expone en este artículo, los beneficios del procedimiento de insolvencia para personas naturales no comerciantes podrían no estarse reportando dada la barrera que imposibilita a muchos deudores insolventes acceder a su trámite; es claro, entonces, que la falta de capacidad económica para iniciar el procedimiento ante un centro de conciliación privado o una notaría, y la inexistencia de centros de conciliación de consultorios jurídicos o de entidades públicas que lo presten niegan esa oportunidad de acceso que enmarca el concepto de efectividad del derecho, por lo que se tiene que, para las personas insolventes en condiciones de vulnerabilidad económica, el régimen no estaría atendiendo a los fines públicos para los cuales se instituyó.

Este panorama plantea una barrera de acceso a los sistemas formalizados de resolución de conflictos por la carencia absoluta de una oferta de servicios de justicia de carácter gratuito para las personas naturales no comerciantes en condiciones de insolvencia pues no habrá accesibilidad real en tanto no existan operadores que tramiten gratuitamente este procedimiento.

Sobre lo anterior, es adecuado indicar que la aptitud del derecho para desencadenar las transformaciones que se esperan de la vigencia de sus normas depende de la dosis de voluntad y de interés con que el poder público las hace cumplir y consuma satisfactoriamente sus efectos. ${ }^{15}$ Luego, entonces, la eficacia del derecho se encuentra radicada en cabeza del Estado, ente idóneo para que las normas se cumplan y se materialicen los efectos para los cuales se crearon. Aquí resulta importante establecer la importancia de las autoridades competentes en la promoción del acceso a la justicia y, particularmente, verificar las acciones que han sido gestionadas por el Ministerio de Justicia y del Derecho para promover, por ejemplo, que los centros de conciliación adscritos a los consultorios jurídicos de las facultades de derecho se habiliten para el trámite del procedimiento de insolvencia de persona natural. A la fecha, esta cartera no cuenta con ningún diagnóstico ni estudio relacionado con el acceso a la justicia para personas naturales no comerciantes ni ha desarrollado una política pública en esta materia.

\section{Factores}

Para identificar los factores que explican por qué en Bogotá ningún centro de conciliación universitario tramita el proceso de insolvencia de persona natural no comerciante se realizaron entrevistas a asesores en materia de conciliación y coordinadores de estos centros de cinco universidades de Bogotá. La ma-

14 Manuel María Zorrilla Ruiz, "Eficacia y efectividad del derecho", Revista Deusto 45 (1) (1997), pp. 173-196. 15 Ibid., 15. 
yoría de los entrevistados expresaron que efectivamente conocen el régimen normativo aplicable a las personas naturales no comerciantes en condiciones de insolvencia, los requisitos que deben cumplir los centros de conciliación para habilitarse en el trámite del procedimiento de insolvencia de persona natural no comerciante, y advierten que, a mediados del mes de julio de 2018, el Ministerio de Justicia generó una alerta sobre este tema, ${ }^{16}$ convocándolos a solicitar la autorización para conocer dichos procedimientos.

La mayoría de entrevistados reconoció que dichos requisitos, en principio, son de factible cumplimiento y no advierten barreras formales que impidan que el centro de conciliación al que están vinculados pida la autorización requerida por parte del Ministerio de Justicia para tramitar esta clase de procedimiento.

Cuando se consultó acerca de los motivos por los cuales su respectivo centro de conciliación no ha solicitado la autorización al Ministerio de Justicia para el trámite de este procedimiento, algunos entrevistados señalaron que la demanda por este servicio podría no ser tan alta, y por estas circunstancias no se han ocupado de este tema. Así mismo, algunos expresaron que existe la posibilidad de eventuales dificultades en el desarrollo de estos procedimientos, dado que se trata de centros de conciliación "pequeños", donde el número de conciliadores es reducido, y consideran que estos procedimientos deberían gestionarlos centros de conciliación robustos que cuenten con los medios y el recurso humano para tramitar estos asuntos.

Las respuestas de los entrevistados coinciden en afirmar que las razones por las cuales no se ha solicitado la autorización para el trámite de este procedimiento no obedecen a motivos o razonamientos que justifiquen que no están interesados o que no se pueda hacer, y señalan que es algo que puede ser revisado y con los avales institucionales requeridos se podría estudiar la posibilidad de hacerlo, pues no hay razones de fondo para no hacerlo, simplemente no se ha pensado esta problemática.

La mayoría de entrevistados coincide en que los recursos y la capacidad institucional siempre es una barrera, y ponen de presente que los consultorios jurídicos son un costo para las facultades de derecho pues no generan ningún tipo de ingreso. Igualmente, señalan que se tendría que evaluar si con la capacidad instalada que se tiene se puede prestar este servicio a la comunidad. Y si se puede, no habría razones para no hacerlo, aunque se requiere un esfuerzo de mayor personal y mayores gastos, lo que podría representar una dificultad porque se trata de un gasto adicional.

16 Según lo reportado por el Ministerio de Justicia y del Derecho de Colombia, en el año 2018, mediante la circular CIR18-0000074-DMA2100, se convocó a los centros de conciliación de facultades de derecho y de entidades públicas de todo el país a solicitar la autorización para conocer de los procedimientos de insolvencia de persona natural no comerciante. 
Sobre si las personas en situación de insolvencia deben ser sujetos de atención de un consultorio jurídico o es mejor que sean dirigidas a instituciones especializadas en estos asuntos, se resalta la importancia de que se encuentren en situación de vulnerabilidad, es decir que se cumplan los propósitos de los consultorios jurídicos: la atención a población vulnerable; pero si se trata de atender casos de personas que, en principio, tienen capacidad económica, ahí podría presentarse una incompatibilidad.

Para algunos de los entrevistados existe una preocupación respecto a cómo el desarrollo de estos procedimientos pudiera estar respondiendo a la presión que ejerce el sistema bancario en el sector de la justicia, y de manera directa en los operadores que prestan servicios legales gratuitos, pues asumir el desarrollo de estos procesos supondría también facilitar herramientas de recuperación de los créditos de acreedores tales como entidades financieras y cooperativas.

Visto lo anterior, las motivaciones por las cuales los centros de conciliación de facultades de derecho no están prestando sus servicios para el trámite del procedimiento de insolvencia son variadas. En todo caso, es importante llamar a la reflexión a estos centros, pues el no adelantamiento del procedimiento de insolvencia contraría los propósitos de estas instituciones ya que, por un lado, se estaría afectando directamente a los usuarios que en condiciones de vulnerabilidad económica concurren a su auxilio para buscar una alternativa legal que permita una solución a su situación financiera, $y$, por otro, no se estaría desarrollando una oportunidad de aprendizaje para los estudiantes.

\section{Conclusiones}

Para concluir, se señala que el derecho debe fijar unas condiciones que permitan responder a las demandas sociales significativas y dignas de atención. En ese sentido, tal como lo indica Hart, la validez del derecho depende de un conjunto de hechos sociales, materializados en las acciones de los miembros de la sociedad. ${ }^{17}$ Innegablemente, Colombia ha atravesado diferentes crisis económicas que han afectado de manera individual a un gran número de asociados, e igualmente estos, en su esfera de actividad económica privada, han incurrido en manejos acéfalos y funestas decisiones de gasto, lo que ha afectado su órbita personal desembocando en crisis financieras.

Frente a la situación de insolvencia de las personas naturales que no son comerciantes, el ordenamiento jurídico nacional contempla un procedimiento de rescate financiero que señala la gratuidad para el adelantamiento de este ante los centros de conciliación de las facultades de derecho. Infortunadamente, y habiendo pasado siete años desde la promulgación de esta ley, ningún centro de conciliación de facultad de derecho de la ciudad de Bogotá está prestando

17 Herbert Hart, The concept of law, New York, Oxford University Press, 1961. 
este servicio, lo que afecta directamente a las personas insolventes en condiciones de vulnerabilidad al no encontrar una oportunidad de alivio a su situación en estos prestadores de servicios legales gratuitos. Sobre lo anterior, y en búsqueda de los factores que explican por qué en la ciudad de Bogotá ningún centro de conciliación de facultad de derecho se ha habilitado para el trámite de estos procedimientos, se identificó que, aun cuando no existen barreras formales, algunos de los operadores de conciliación de facultades de derecho de la ciudad consideran que este procedimiento pudiera ser gestionado por centros de conciliación que cuenten con el recurso humano y físico adecuado para su desarrollo o que se tendría que verificar si existen las condiciones físicas y humanas para su prestación. Se señala que a 2019, el Ministerio de Justicia no había desarrollado una política pública en materia de acceso a la justicia para personas naturales no comerciantes en situación de insolvencia. En este mismo sentido, no ha desplegado una estrategia para que los centros de conciliación de las facultades de derecho se habiliten para el trámite del procedimiento de insolvencia de persona natural no comerciante.

\section{Bibliografía}

Alemán Monterreal, Ana, La insolvencia: una cuestión de terminología jurídica, Madrid, Andavira Editora, 2010.

AA.VV., Pobreza: un glosario internacional, Buenos Aires, Clacso, 2009.

Baratz, Morton S. y William Grigsby, Thoughts on poverty and its elimination, Cambridge, Cambridge University Press, 1972.

Bonilla Maldonado, Daniel, Abogados y justicia social: derecho de interés público y clínicas jurídicas, Bogotá, Ediciones Uniandes, Siglo del Hombre Editores, 2018.

Guataquí Roa, Juan Carlos, Juan Pablo Baquero, Iván Daniel Jaramillo, Johan Andrey Ortega y Marlon Salazar, Informe 4: Panorama de la negociación del salario mínimo 2018, Bogotá, Observatorio Laboral de la Universidad del Rosario, 2018.

Hart, Herbert. The concept of law, New York: Oxford University Press, 1961.

Portafolio, "Aún hay 3,5 millones de personas en pobreza extrema", Portafolio, 2018, en https://www.portafolio.co/economia/aun-hay-3-5-millones-depersonas-en-pobreza-extrema-522423

Spicker, Paul, Sonia Álvarez Leguizamón y David Gordon, Pobreza: un glosario internacional, Buenos Aires, Clacso, 2009.

Zorrilla, Manuel María, "Eficacia y efectividad del derecho", Revista Deusto 45

(1) (1997). DOI: http://dx.doi.org/10.18543/ed-45(1)-1997pp173-196 